\title{
Compact finite difference method to numerically solving a stochastic fractional advection-diffusion equation
}

\author{
N.H. Sweilam ${ }^{1 *}$, D.M. El-Sakout ${ }^{1}$ and M.M. Muttardi
}

\section{"Correspondence:}

nsweilam@sci.cu.edu.eg

'Department of Mathematics,

Faculty of Science, Cairo University,

Giza, Egypt

\section{Springer}

\begin{abstract}
In this paper, a stochastic space fractional advection diffusion equation of Itô type with one-dimensional white noise process is presented. The fractional derivative is defined in the sense of Caputo. A stochastic compact finite difference method is used to study the proposed model numerically. Stability analysis and consistency for the stochastic compact finite difference scheme are proved. Two test examples are given to test the performance of the proposed method. Numerical simulations show that the results obtained are compatible with the exact solutions and with the solutions derived in the literature.
\end{abstract}

MSC: $65 \mathrm{M} 70 ; 65 \mathrm{~N} 35 ; 35 \mathrm{C} 10 ; 42 \mathrm{C} 10$

Keywords: Stochastic fractional advection-diffusion equation; Stochastic compact difference scheme; Stability analysis of a stochastic model; Caputo fractional derivative

\section{Introduction}

Many physical phenomena are simulated using mathematical models that lead to partial differential deterministic equations (PDEs). Due to a lack of data on parameters and initial data, the behavior of the system might be far away from the ideal deterministic representation. To counter this lack of information and make a system explanation more practical, one adds random inputs that can be random variables or stochastic processes. This leads to stochastic partial differential equations (SPDEs).

SPDEs are used in multiple fields including computational mathematics, electronics, statistical mechanics, theoretical physics, theoretical psychology, advanced chemical reaction analysis, fluid dynamics, hydrology, and mathematical finance. In certain types of SPDEs, analytical approaches, such as Prato et al. and Gyongy et al. [1-4], can be provided. For the numerical simulations of SPDEs, Allen et al., Walsh and Deb et al. [5-7] used the finite element approximation. In [8] high-resolution finite volume methods are used to solve SPDE. Roth [9] used an explicit finite difference method to approximate a solution of some stochastic hyperbolic equations. Soheili et al. [10] presented two methods for solving linear parabolic SPDE's based on Saulyev method and a high order finite

(c) The Author(s) 2020. This article is licensed under a Creative Commons Attribution 4.0 International License, which permits use, sharing, adaptation, distribution and reproduction in any medium or format, as long as you give appropriate credit to the original author(s) and the source, provide a link to the Creative Commons licence, and indicate if changes were made. The images or other third party material in this article are included in the article's Creative Commons licence, unless indicated otherwise in a credit line to the material. If material is not included in the article's Creative Commons licence and your intended use is not permitted by statutory regulation or exceeds the permitted use, you will need to obtain permission directly from the copyright holder. To view a copy of this licence, visit http://creativecommons.org/licenses/by/4.0/. 
difference scheme. Kamrani and Hosseini [11] reported explicit and implicit finite difference methods for general SPDE. Kamrani et al. and Ashyralyev [12, 13] used spectral method for spatial variable discretization and solved the resulting system via the stochastic Runge-Kutta method.

Integer order differential equations might not be capable of explaining the experimental and field measurement data, as an alternate approach, the differential fractional-order equation (FDE) is introduced [14-22]. Models described by FDEs are now being widely used, and the theory of fractional calculus is a useful mathematical tool for applied science [23-26]. Pardisi et al. and Baeumer et al. [27, 28] have developed models in fractional order containing various concepts. Fractional derivatives are adapted to model diffusion by stochastic fractional differential equations (SFDEs).

In SFDEs the particles distributed differently than predicted by the classical Brownian model of motion are expected [29-31]. The impetus to considering such equations derives from the idea that there is some ambiguity in other science processes that have been modeled by fractional differential equations, and we require SFDE solutions to test more precise solutions.

Advection-diffusion equation (ADE) explains the transfer of mass, heat, or energy due to the combination of advection and diffusion effect. In addition, ADE describes other natural processes such as air contamination, groundwater contamination, the distribution of chemical solutes, the infiltration of seawater and thermal penetration of river systems, to explain the time and space differences of particle behavior [32,33]. Fractional advectiondiffusion equations (FADEs) are used to model transportation through a porous medium with passive tracers provided by fluid movement. Liu et al. [34] found and used vector transformation to solve the time-fractional ADE. Huang and Liu [35] obtained space timefractional ADE solution, Meerschaert et al. [36] proposed functional computational methods for solving the space fractional ADE solution.

Stochastic simulations include evidence on the statistical features of particle behavior [37]. Such equations are very helpful because moving sediments experience significant variations, rendering it impossible to calculate mean values. Among these stochastic models, the most common approach consists of random walk models [38]. In [39] Soheili and Bishehniasar considered the approximation of stochastic advection-diffusion equation using compact finite difference technique and investigated their numerical results.

In this paper, we introduced a stochastic fractional advection-diffusion equation (SFADE), which can be considered as a generalization of the classical ADE with a one-dimensional multiplicative white noise process, replacing the second and first-order space derivatives with the Caputo fractional-order derivative $\alpha \in(1,2]$ and $\beta \in(0,1]$, respectively. Our goal in the current research is to approximate the solution of SFADE with respect to an $R^{1}$ valued Wiener process $\left(W(t), F_{t}\right)_{t \in[0, T]}$ defined on a complete probability space $(\Omega, F, P)$ [9] adapted to standard filtration $\left(F_{t}\right)_{t \in[0, T]}$. The efficiency of the stochastic compact finite difference (SCFD) process for the SFADE is shown and its key qualifications are examined.

The paper is structured as follows: The considered SFADE and some mathematical tools are given in Sect. 2. SCFD scheme to approximate the solution of SFADE is introduced in Sect. 3. In Sect. 4 we investigate the consistency and stability analysis of the proposed method. Numerical simulations are given in Sect. 5. Finally, the conclusions are drawn in Sect. 6 . 


\section{Preliminaries and fundamentals}

In the first part of this section, we introduce the stochastic fractional advection-diffusion equation (SFADE) with Dirichlet boundary conditions, then we recall some mathematical tools.

Consider the SFADE:

$$
\begin{aligned}
& u_{t}(x, t)=\rho D_{x}^{\alpha} u(x, t)-\gamma D_{x}^{\beta} u(x, t)+\sigma u(x, t) \dot{W}(t), \\
& 0<t \leq T, \quad 0<x<X, \\
& u(x, 0)=u_{0}(x), \quad 0<x<X, \\
& u(0, t)=f_{1}(t), \quad u(X, t)=f_{2}(t), \quad 0<t \leq T,
\end{aligned}
$$

where $u$ is the quantity being transported, $\rho>0$ is the dispersion/diffusion coefficient, $\gamma>$ 0 is the advection coefficient, and $\sigma>0$ is noise intensity; $T, X, \rho, \gamma$, and $\sigma$ are constants and $D_{x}^{\alpha}, D_{x}^{\beta}$ are the Caputo space fractional derivatives of order $\alpha, \beta$, respectively, $1<\alpha \leq 2$ and $0<\beta \leq 1, f_{1}(t)$ and $f_{2}(t)$ are continuous given functions. $W(t)$ is a one-dimensional standard Wiener process such that the white noise $\Delta W(t)$ is a random variable generated from Gaussian distribution with zero mean and standard deviation $\Delta t$, and we will define it later[40]. We need the following assumption in sequel.

Assumption 1 We assume that $u \in \mathbf{C}^{6,1}(\Omega), \Omega=[0, X] \times[0, T]$, to ascertain the sufficient smoothness conditions for the existence of the fractional derivatives as well as the validity of the stochastic compact finite difference scheme, where $\mathbf{C}^{6,1}$ is the well-known space of all $u$ such that $\frac{\partial^{6} u}{\partial x^{6}}$ and $\frac{\partial u}{\partial t}$ are continuous.

Numerically, finite difference methods have vast applications $[9,10]$. These schemes discretize continuous space and time into an evenly distributed grid system, and the values of the state variables are evaluated at each node of the grid. Introduce the grid points $x_{i}=$ $i h$, for $h=\frac{X}{N}$ and $i=0,1, \ldots, N . t_{n}=n \Delta t$, where $\Delta t=\frac{T}{M}$ and $n=0,1, \ldots, M$, where $N$ and $M$ are the total number of grid points for the space and time discretizations, respectively. Let $u_{i}(t)$ be the finite difference approximation to $\left[u\left(t, x_{1}\right), \ldots, u\left(t, x_{N-1}\right)\right]^{T}$.

To discretize in time, we apply the Euler-Maruyama method with time step $\Delta t>0$. This method gives approximations $u_{i}^{n}$ to $\left[u\left(t_{n}, x_{1}\right), \ldots, u\left(t_{n}, x_{N-1}\right)\right]^{T}$.

Definition 1 (Euler-Maruyama scheme, [9]) For the d-dimensional SDE of Itô type

$$
\left\{\begin{array}{l}
d u(t)=f(t, u(t)) d t+g(t, u(t)) d W(t), \\
t \geq 0, \quad u(0)=u_{0} \in \mathbb{R}^{d},
\end{array}\right.
$$

where $f, g: \mathbb{R}^{d} \rightarrow \mathbb{R}^{d}$, the Euler-Maruyama scheme for computing approximations $u_{i}^{n}$ takes the form

$$
u_{i}^{n+1}=u_{i}^{n}+f\left(u_{i}^{n}\right) \Delta t+g\left(u_{i}^{n}\right) \Delta W_{n},
$$

where $\Delta W_{n}=W\left(t_{n+1}\right)-W\left(t_{n}\right)=W((n+1) \Delta t)-W((n) \Delta t)$ and $\Delta W_{n} \sim \mathcal{N}(0, \Delta t)$. 
There are different ways to define fractional derivatives, the most commonly used are the Grünwald-Letnikov derivative, the Riemann-Liouville derivative, and the Caputo derivative [41], [48-52].

Definition 2 The Caputo fractional derivative of order $\mu$ is defined as [42]

$$
D^{\mu} f(x)=\frac{1}{\Gamma(m-\mu)} \int_{0}^{x}(x-t)^{m-\mu-1} \frac{d^{m}}{d t^{m}} f(t) d t, \quad m-1<\mu<m, x>0,
$$

where $\mu$ is a fractional number and $m$ is an integer number.

Note 3 ([42]) Assumption 1 ascertains the existence of the fractional derivatives $D_{x}^{\alpha}, D_{x}^{\beta}$.

Substituting Eq. (2) in Eq. (1), we obtain

$$
\begin{aligned}
u_{t}\left(x_{i}, t\right)= & \frac{\rho}{\Gamma(2-\alpha)} \int_{0}^{x_{i}}\left(x_{i}-\zeta\right)^{1-\alpha} \frac{\partial^{2}}{\partial \zeta^{2}} u(\zeta, t) d \zeta \\
& -\frac{\gamma}{\Gamma(1-\beta)} \int_{0}^{x_{i}}\left(x_{i}-\zeta\right)^{-\beta} \frac{\partial}{\partial \zeta} u(\zeta, t) d \zeta+\sigma u\left(x_{i}, t\right) \dot{W}(t), \\
u_{t}\left(x_{i}, t\right)= & \frac{\rho}{\Gamma(2-\alpha)} \int_{0}^{x_{i}}\left(x_{i}-\zeta\right)^{1-\alpha} \frac{\partial^{2}}{\partial \zeta^{2}} u(\zeta, t) d \zeta \\
& -\frac{\gamma}{\Gamma(1-\beta)} \int_{0}^{x_{i}}\left(x_{i}-\zeta\right)^{-\beta} \frac{\partial}{\partial \zeta} u(\zeta, t) d \zeta+\sigma u\left(x_{i}, t\right) \dot{W}(t),
\end{aligned}
$$

by disctretizing the integrals, we get

$$
\begin{aligned}
= & \frac{\rho}{\Gamma(2-\alpha)} \sum_{r=0}^{i-1} \int_{r h}^{(r+1) h}\left(x_{i}-\zeta\right)^{1-\alpha} \frac{\partial^{2}}{\partial \zeta^{2}} u(\zeta, t) d \zeta \\
& -\frac{\gamma}{\Gamma(1-\beta)} \sum_{r=0}^{i-1} \int_{r h}^{(r+1) h}\left(x_{i}-\zeta\right)^{-\beta} \frac{\partial}{\partial \zeta} u(\zeta, t) d \zeta+\sigma u\left(x_{i}, t\right) \dot{W}(t) .
\end{aligned}
$$

\section{The numerical method of SFADE}

In this section, we introduce a stochastic compact finite difference method (SCFDM) for solving the stochastic fractional advection-diffusion equation (SFADE).

\subsection{Stochastic compact finite difference approximation}

Compact finite difference approximates $u_{x}$ and $u_{x x}$ to order of accuracy higher than two [43]. Here $u_{x}$ and $u_{x x}$ are approximated by fourth order approximation with truncation error $\mathcal{O}\left(h^{4}\right)$.

In the explicit compact method, the time and space derivatives in the SFADE are approximated in the following form [43]:

$$
\begin{aligned}
& u_{x x}(i h, n \Delta t)=\frac{\frac{-1}{12} u_{i-2}^{n}+\frac{4}{3} u_{i-1}^{n}-\frac{5}{2} u_{i}^{n}+\frac{4}{3} u_{i+1}^{n}-\frac{1}{12} u_{i+2}^{n}}{h^{2}}+\mathcal{O}\left(h^{4}\right), \\
& u_{x}(i h, n \Delta t)=\frac{\frac{1}{12} u_{i-2}^{n}-\frac{4}{3} u_{i-1}^{n}+\frac{4}{3} u_{i+1}^{n}-\frac{1}{12} u_{i+2}^{n}}{h}+\mathcal{O}\left(h^{4}\right) .
\end{aligned}
$$


The stochastic partial differential equation Eq. (1) should be read as

$$
d u(x, t)=\left[\rho D_{x}^{\alpha} u(x, t)-\gamma D_{x}^{\beta} u(x, t)\right] d t+\sigma u(x, t) d W(t)
$$

which is equivalent to

$$
d u=\varphi(u) d t+\psi(u) d W(t)
$$

where the stochastic integral is the usual Itô-integral. $\varphi(u)=\rho D_{x}^{\alpha} u(x, t)-\gamma D_{x}^{\beta} u(x, t)$ and $\psi(u)=\sigma u(x, t)$ are Lipschitz-continuous functions in $u(x, t), u_{x}(x, t)$, and $u_{x x}(x, t)$.

According to [9], a solution of stochastic differential equation is a random field $(u(x, t))_{x \in \mathbb{R}, t \in[0, T]}$ with the following properties:

- $(u(x, t))_{x \in \mathbb{R}, t \in[0, T]}$ is an adapted process to the standard filtration $\left(F_{t}\right)_{t \in[0, T]}$ for every $x \in \mathbb{R}$.

- There exists continuous $\frac{\partial^{2} u(x, t)}{\partial x^{2}}$ for every $t \in[0, T]$ with probability 1 .

- $u(\cdot, t)$ is $L^{2}(\mathbb{R})$ valued.

- $u(x, 0)=u_{0}(x) \in L^{2}(\mathbb{R})$.

- Equation $d u=\varphi(u) d t+\psi(u) d W(t)$ holds for all $t \in[0, T]$ and $x \in \mathbb{R}$ with probability 1 .

Assumption (1) guarantees the existence of a unique solution of the last equation ([44], Theorem 8.18, p. 335).

Now, by using the Euler-Maruyama method and substituting Eqs. (4) in (3), we have

$$
\begin{aligned}
u_{i}^{n+1}= & u_{i}^{n}+\frac{\Delta t \rho h^{-\alpha}}{\Gamma(3-\alpha)} \sum_{r=0}^{i-1}\left(-1 / 12 u_{i-r-2}^{n}+4 / 3 u_{i-r-1}^{n}-5 / 2 u_{i-r}^{n}+4 / 3 u_{i-r+1}^{n}-1 / 12 u_{i-r+2}^{n}\right) \\
& \times\left[(i-r+1)^{2-\alpha}-(i-r)^{2-\alpha}\right] \\
& -\frac{\Delta t \gamma h^{-\beta}}{\Gamma(2-\beta)} \sum_{r=0}^{i-1}\left(1 / 12 u_{i-r-2}^{n}-4 / 3 u_{i-r-1}^{n}+4 / 3 u_{i-r+1}^{n}-1 / 12 u_{i-r+2}^{n}\right) \\
& \times\left[(i-r+1)^{1-\beta}-(i-r)^{1-\beta}\right]+\sigma u_{i}^{n} \Delta W_{n} .
\end{aligned}
$$

Let $i-r=k$,

$$
\begin{aligned}
u_{i}^{n+1}= & u_{i}^{n}+\frac{\Delta t \rho h^{-\alpha}}{\Gamma(3-\alpha)} \sum_{k=1}^{i}\left(-1 / 12 u_{i-k-2}^{n}+4 / 3 u_{i-k-1}^{n}-5 / 2 u_{i-k}^{n}+4 / 3 u_{i-k+1}^{n}-1 / 12 u_{i-k+2}^{n}\right) \\
& \times\left[(k)^{2-\alpha}-(k-1)^{2-\alpha}\right] \\
& -\frac{\Delta t \gamma h^{-\beta}}{\Gamma(2-\beta)} \sum_{k=1}^{i}\left(1 / 12 u_{i-k-2}^{n}-4 / 3 u_{i-k-1}^{n}+4 / 3 u_{i-k+1}^{n}-1 / 12 u_{i-k+2}^{n}\right) \\
& \times\left[(k)^{1-\beta}-(k-1)^{1-\beta}\right]+\sigma u_{i}^{n} \Delta W_{n} .
\end{aligned}
$$

Let

$$
b^{\alpha}=\frac{\rho \Delta t}{h^{\alpha} \Gamma(3-\alpha)}, \quad b^{\beta}=\frac{\gamma \Delta t}{h^{\beta} \Gamma(2-\beta)},
$$




$$
g_{k}^{\alpha}=(k)^{2-\alpha}-(k-1)^{2-\alpha}, \quad g_{k}^{\beta}=(k)^{1-\beta}-(k-1)^{1-\beta} .
$$

Taking the boundary conditions into consideration ([43], p. 39, HW1.5.12), we have

$$
\begin{aligned}
u_{i}^{n+1}= & u_{i}^{n} \\
& +b^{\alpha} \sum_{k=1}^{i} g_{k}^{\alpha}\left(-1 / 12 u_{i-k-2}^{n}+4 / 3 u_{i-k-1}^{n}-5 / 2 u_{i-k}^{n}+4 / 3 u_{i-k+1}^{n}-1 / 12 u_{i-k+2}^{n}\right) \\
& -b^{\beta} \sum_{k=1}^{i} g_{k}^{\beta}\left(1 / 12 u_{i-k-2}^{n}-4 / 3 u_{i-k-1}^{n}+4 / 3 u_{i-k+1}^{n}-1 / 12 u_{i-k+2}^{n}\right)+\sigma u_{i}^{n} \Delta W_{n} .
\end{aligned}
$$

Equation (6), together with boundary conditions, yields the following linear system of equations:

$$
\mathbf{U}^{n+1}=\mathbf{U}^{n}+\mathbf{A U}^{n}-\mathbf{B} \mathbf{U}^{n}+\sigma \mathbf{U}^{n} \Delta W_{n}
$$

where $\mathbf{U}^{n}=\left(u_{1}^{n}, u_{2}^{n}, \ldots, u_{N-1}^{n}\right)^{T}$, and $\mathbf{A}_{N-1 \times N-1}=\left(a_{j k}\right)$ and $\mathbf{B}_{N-1 \times N-1}=\left(b_{j k}\right)$ are the coefficient matrices with the following elements:

$$
a_{j k}= \begin{cases}0, & k \geq j+3 ; \\ -1 / 12 b^{\alpha}, & k=j+2 ; \\ b^{\alpha}\left(4 / 3-1 / 12 g_{1}^{\alpha}\right), & k=j+1 ; \\ b^{\alpha}\left(-5 / 2+4 / 3 g_{1}^{\alpha}-1 / 12 g_{2}^{\alpha}\right), & k=j=3, \ldots, N-1 ; \\ b^{\alpha}\left(4 / 3-5 / 2 g_{1}^{\alpha}+4 / 3 g_{2}^{\alpha}-1 / 12 g_{3}^{\alpha}\right), & k=j-1 ; \\ b^{\alpha}\left(-1 / 12 g_{j-k-2}^{\alpha}+4 / 3 g_{j-k-1}^{\alpha}\right. & \\ \left.\quad-5 / 2 g_{j-k}^{\alpha}+4 / 3 g_{j-k+1}^{\alpha}-1 / 12 g_{j-k+2}^{\alpha}\right), & k \leq j-2 ;\end{cases}
$$

for $k=3,4, \ldots, N-1$ and $j=1,2, \ldots, N-1$, while

$$
\begin{aligned}
& a_{11}=-5 / 2 b^{\alpha}, \quad a_{21}=b^{\alpha}\left(\frac{4}{3}-\frac{5}{2} g_{1}^{\alpha}\right), \\
& a_{j 1}=b^{\alpha}\left(-1 / 12 g_{j-3}^{\alpha}+4 / 3 g_{j-2}^{\alpha}-5 / 2 g_{j-1}^{\alpha}\right), \quad 3 \leq j \leq N-1, \\
& a_{12}=\frac{4}{3} b^{\alpha}, \quad a_{22}=b^{\alpha}\left(-5 / 2+4 / 3 g_{1}^{\alpha}\right), \\
& a_{32}=b^{\alpha}\left(4 / 3-5 / 2 g_{1}^{\alpha}+4 / 3 g_{2}^{\alpha}\right), \\
& a_{j 2}=b^{\alpha}\left(-1 / 12 g_{j-4}^{\alpha}+4 / 3 g_{j-3}^{\alpha}-5 / 2 g_{j-2}^{\alpha}+4 / 3 g_{j-1}^{\alpha}\right), \quad 4 \leq j \leq N-1 . \\
& b_{j k}= \begin{cases}0, & k \geq j+3 ; \\
-1 / 12 b^{\beta}, & k=j+2 ; \\
b^{\beta}\left(4 / 3-1 / 12 g_{1}^{\beta}\right), & k=j=3, \ldots, N-1 ; \\
b^{\beta}\left(4 / 3 g_{1}^{\beta}-1 / 12 g_{2}^{\beta}\right), & k=j-1 ; \\
b^{\beta}\left(-4 / 3+4 / 3 g_{2}^{\beta}-1 / 12 g_{3}^{\alpha}\right), & \\
b^{\beta}\left(1 / 12 g_{j-k-2}^{\beta}-4 / 3 g_{j-k-1}^{\beta}\right. & \\
\left.+4 / 3 g_{j-k+1}^{\beta}-1 / 12 g_{j-k+2}^{\beta}\right), & k \leq j-2 ;\end{cases}
\end{aligned}
$$


for $k=3,4, \ldots, N-1$ and $j=1,2, \ldots, N-1$, while

$$
\begin{aligned}
& b_{11}=0, \quad b_{21}=-4 / 3 b^{\beta}, \quad b_{31}=b^{\beta}\left(1 / 12-4 / 3 g_{1}^{\beta}\right), \\
& b_{j 1}=b^{\beta}\left(1 / 12 g_{j-3}^{\beta}-4 / 3 g_{j-2}^{\beta}\right), \quad 4 \leq j \leq N-1, \\
& b_{12}=4 / 3 b^{\beta}, \quad b_{22}=4 / 3 b^{\beta} g_{1}^{\beta}, \quad b_{32}=b^{\beta}\left(-4 / 3+4 / 3 g_{2}^{\beta}\right), \\
& b_{24}=b^{\beta}\left(1 / 12-4 / 3 g_{1}^{\beta}+4 / 3 g_{3}^{\beta}\right), \\
& b_{j 2}=b^{\beta}\left(1 / 12 g_{j-4}^{\beta}-4 / 3 g_{j-3}^{\beta}+4 / 3 g_{j-1}^{\beta}\right), \quad 5 \leq j \leq N-1 .
\end{aligned}
$$

In a component from Eq. (7), we have

$$
U_{m}^{n+1}=U_{m}^{n}+\sum_{k} A_{m k} U_{k}^{n}-\sum_{k} B_{m k} U_{k}^{n}+\sigma \sqrt{\Delta t} V_{n} U_{m}^{n}
$$

in which $m=1, \ldots, N-1$, where $V_{n}$ is a normally distributed random variable with zero mean and unit variance, i.e, $\mathcal{N}(0,1)$ random variable [45].

\section{Stability analysis of the stochastic scheme}

In this section, some basic stochastic version definitions are given for the main concepts of deterministic finite difference schemes. These concepts are extremely important for the results of stochastic difference schemes (SDS) to converge to the solution of the stochastic differential equations. To get a higher degree of generality, we consider a stochastic partial differential equation (SPDE), say

$$
\mathcal{L} v(x, t)=G(x, t), \quad x \in \mathbb{R}, t \geq 0,
$$

with an initial condition $v(x, 0)=v_{0}$, where $\mathcal{L}$ denotes the differential operator and $G \in L^{2}(\mathbb{R})$ is an inhomogeneity. Assuming $u_{i}^{n}$ is a solution of this problem, which is approximated by a stochastic compact finite difference scheme denoted by $\mathcal{L}_{i}^{n}$, and applying the stochastic scheme to the SPDE, we have $\mathcal{L}_{i}^{n} u_{i}^{n}=G_{i}^{n}$, whereby $G_{i}^{n}$ is the approximation of the inhomogeneity. As before, $n$ corresponds to the time step and $i$ to the spatial mesh point. For the following definitions [9], let us introduce, for the sequence $\mathbf{x}=\left\{\ldots, x_{-1}, x_{0}, x_{1}, \ldots\right\}$, the $\ell_{2, \Delta x}$-norm $|\mathbf{x}|_{2, \Delta x}=\sqrt{\sum_{i=-\infty}^{\infty}\left|x_{i}\right|^{2} \Delta x}$ and the sup-norm $|\mathbf{x}|_{\infty}=\sqrt{\sup _{i}\left|x_{i}\right|^{2}}$.

Definition 4 (Consistency of a stochastic difference scheme, [9]) For a stochastic compact finite difference scheme $\mathcal{L}_{i}^{n}$, a stochastic difference scheme $\mathcal{L}_{i}^{n} u_{i}^{n}=G_{i}^{n}$ is pointwise consistent with the stochastic partial differential equation $\mathcal{L} v=G$ at a point $(x, t)$ if, for any continuously differentiable function $\Phi=\Phi(x, t)$, in the mean square

$$
\mathbb{E}\left\|\left.(\mathcal{L} \Phi-G)\right|_{i} ^{n}-\left[\mathcal{L}_{i}^{n} \Phi(i \Delta x, n \Delta t)-G_{i}^{n}\right]\right\|^{2} \rightarrow 0
$$

as $\Delta x \rightarrow 0, \Delta t \rightarrow 0$ and $(i \Delta x,(n+1) \Delta t)$ converges to $(x, t)$.

Definition 5 (Stability of a stochastic difference scheme, [9]) A stochastic difference scheme is said to be stable with respect to a norm in the mean square if there exist some 
positive constants $\overline{\Delta x_{0}}$ and $\overline{\Delta t_{0}}$ and non-negative constants $K$ and $\theta$ such that

$$
\mathbb{E}\left\|u^{n+1}\right\|^{2} \leq K e^{\theta t} E\left\|u^{0}\right\|^{2}
$$

for all

$$
0 \leq t=(n+1) \Delta t, \quad 0 \leq \Delta x \leq \overline{\Delta x_{0}}, \quad 0 \leq \Delta t \leq \overline{\Delta t_{0}},
$$

where $u^{n+1}$ is the infinite dimensional vector:

$$
u^{n+1}=\left(\ldots, u_{i-2}^{n+1}, u_{i-1}^{n+1}, u_{i}^{n+1}, u_{i+2}^{n+1}, u_{i+1}^{n+1}, \ldots\right)^{T}
$$

Note that the norm used above was not specified. This is done in this manner because in different situations different norms will be used.

Remark 6 By using Fourier analysis, Von Neumann [46] introduces a method to prove stability and gives a necessary and sufficient condition for the stability of deterministic finite difference schemes.

Assuming $\hat{u}^{n+1}$ is the Fourier transformation of $u^{n+1}$, the Fourier-inversion formula gives

$$
u_{m}^{n+1}=\frac{1}{\sqrt{2 \pi}} \int_{-\pi / \Delta x}^{\pi / \Delta x} e^{i m \Delta x \xi} \hat{u}^{n+1}(\xi) d \xi
$$

where

$$
\hat{u}^{n+1}=\frac{1}{\sqrt{2 \pi}} \sum_{m=-\infty}^{m=\infty} e^{-i m \Delta x \xi} u_{m}^{n+1} \Delta x,
$$

$\xi$ is a real variable. Substituting in a stochastic difference scheme, we have

$$
\hat{u}^{n+1}(\xi)=g(\Delta x \xi, \Delta t, \Delta x) \hat{u}^{n}(\xi)
$$

where $g(\Delta x \xi, \Delta t, \Delta x)$ is the amplification factor of the stochastic difference scheme. We get the following necessary and sufficient condition for a scheme's stability via its amplification factor [9]:

$$
\mathbb{E}|g(\Delta x \xi, \Delta t, \Delta x)|^{2} \leq 1+K \Delta t
$$

\subsection{Stability analysis of SCFD scheme}

Let us prove now that the scheme in Eq. (7) fulfills the conditions of the above definitions with $\Delta x=h$.

Theorem 7 If $\left|\left(1+\sum_{k} A_{m k} e^{i(k-m) \Delta x \xi}-\sum_{k} B_{m k} e^{i(k-m) \Delta x \xi}\right)\right| \leq 1$, then the SCFD scheme Eq. (7) is conditionally stable according to the Fourier-transformation analysis for the SFADE. 
Proof Using Eq. (11), Eq. (12) and substituting in Eq. (8), we have

$$
\hat{U}^{n+1}(\xi)=\hat{U}^{n}(\xi)+\sum_{k} A_{m k} \hat{U}^{n}(\xi) e^{i(k-m) \Delta x \xi}-\sum_{k} B_{m k} \hat{U}^{n}(\xi) e^{i(k-m) \Delta x \xi}+\sigma \sqrt{\Delta t} V_{n} \hat{U}^{n}(\xi)
$$

then we get

$$
\hat{U}^{n+1}(\xi)=\left(1+\sum_{k} A_{m k} e^{i(k-m) \Delta x \xi}-\sum_{k} B_{m k} e^{i(k-m) \Delta x \xi}+\sigma \sqrt{\Delta t} V_{n}\right) \hat{U}^{n}(\xi) .
$$

Therefore, the amplification factor of the SCFD scheme is

$$
\begin{aligned}
& g(\Delta x \xi, \Delta t, \Delta x)=\left(1+\sum_{k} A_{m k} e^{i(k-m) \Delta x \xi}-\sum_{k} B_{m k} e^{i(k-m) \Delta x \xi}+\sigma \sqrt{\Delta t} V_{n}\right) . \\
& \mathbb{E}|g(\Delta x \xi, \Delta t, \Delta x)|^{2}=\mathbb{E}\left|1+\sum_{k} A_{m k} e^{i(k-m) \Delta x \xi}-\sum_{k} B_{m k} e^{i(k-m) \Delta x \xi}+\sigma \sqrt{\Delta t} V_{n}\right|^{2},
\end{aligned}
$$

then we get

$$
\begin{aligned}
& \mathbb{E}|g(\Delta x \xi, \Delta t, \Delta x)|^{2} \\
& =\mathbb{E}\left|1+\sum_{k} A_{m k} e^{i(k-m) \Delta x \xi}-\sum_{k} B_{m k} e^{i(k-m) \Delta x \xi}\right|^{2}+\mathbb{E}\left|\sigma \sqrt{\Delta t} V_{n}\right|^{2} \\
& \quad+2 \mathbb{E}\left|\left(1+\sum_{k} A_{m k} e^{i(k-m) \Delta x \xi}-\sum_{k} B_{m k} e^{i(k-m) \Delta x \xi}\right) \times\left(\sigma \sqrt{\Delta t} V_{n}\right)\right|
\end{aligned}
$$

Due to independence of the Wiener process, with $E\left[V_{n}\right]=0$ and $E\left[V_{n}^{2}\right]=1$, we obtain

$$
\mathbb{E}|g(\Delta x \xi, \Delta t, \Delta x)|^{2}=\mathbb{E}\left|1+\sum_{k} A_{m k} e^{i(k-m) \Delta x \xi}-\sum_{k} B_{m k} e^{i(k-m) \Delta x \xi}\right|^{2}+\mathbb{E}|\sigma \sqrt{\Delta t}|^{2} .
$$

Then, if $\left|\left(1+\sum_{k} A_{m k} e^{i(k-m) \Delta x \xi}-\sum_{k} B_{m k} e^{i(k-m) \Delta x \xi}\right)\right| \leq 1$ and $\sigma^{2} \leq K$, we have

$$
\mathbb{E}|g(\Delta x \xi, \Delta t, \Delta x)|^{2} \leq 1+K \Delta t
$$

Then, according to the Fourier-transformation analysis, SCFD scheme is stable if

$$
\mathbb{E}|g(\Delta x \xi, \Delta t, \Delta x)|^{2} \leq 1+K \Delta t
$$

Before proving the following theorem, we define the following:

$$
\begin{aligned}
& \sup _{m}\left|\sum_{k} A_{m k}\right|=\sum_{k} A_{l k}, \\
& \sup _{m}\left|\sum_{k} B_{m k}\right|=\sum_{k} B_{q k} .
\end{aligned}
$$


Theorem 8 If $\left(1+\sum_{k} A_{l k}-\sum_{k} B_{q k}\right) \leq 1$, then the SCFD scheme Eq. (7) with $(n+1) \Delta t=t$ is conditionally stable in the mean square with respect to the norm $|\cdot|_{\infty}=\sqrt{\sup _{i}|\cdot|^{2}}$ for the SEFKE.

Proof Applying $E|\cdot|^{2}$ to Eq. (8) and using the independence of the Wiener increment, with $E\left[V_{n}\right]=0$ and $E\left[V_{n}^{2}\right]=1$, we obtain

$$
\begin{aligned}
& E\left|U_{m}^{n+1}\right|^{2}=E\left[U_{m}^{n}+\sum_{k} A_{m k} U_{k}^{n}-\sum_{k} B_{m k} U_{k}^{n}+\sigma \sqrt{\Delta t} V_{n} U_{m}^{n}\right]^{2}, \\
& E\left|U_{m}^{n+1}\right|^{2}=E\left|U_{m}^{n}+\sum_{k} A_{m k} U_{k}^{n}-\sum_{k} B_{m k} U_{k}^{n}\right|^{2}+\sigma^{2} \Delta t E\left|U_{m}^{n}\right|^{2} .
\end{aligned}
$$

Therefore by Eq. (13) and Eq. (14)

$$
\begin{aligned}
& E\left|U_{m}^{n+1}\right|^{2} \leq\left[\left[1+\sum_{k} A_{l k}-\sum_{k} B_{q k}\right]^{2}+\sigma^{2} \Delta t\right] \sup _{m} E\left|U_{m}^{n}\right|^{2} \\
& \sup _{m} E\left|U_{m}^{n+1}\right|^{2} \leq\left[S C^{2}+\sigma^{2} \Delta t\right] \sup _{m} E\left|U_{m}^{n}\right|^{2}
\end{aligned}
$$

where

$$
S C=1+\sum_{k} A_{l k}-\sum_{k} B_{q k} .
$$

This holds for all $m$, and therefore we get

$$
\sup _{m} E\left|U_{m}^{n+1}\right|^{2} \leq\left[S C^{2}+\sigma^{2} \Delta t\right]^{n+1} \sup _{m} E\left|U_{m}^{0}\right|^{2}
$$

Obviously, if $S C \leq 1$, then with $(n+1) \Delta t=t$ we have

$$
\begin{aligned}
& \sup _{m} E\left|U_{m}^{n+1}\right|^{2} \leq\left[1+\sigma^{2} \frac{t}{t+1}\right]^{n+1} \sup _{m} E\left|U_{m}^{0}\right|^{2}, \\
& E\left\|U_{m}^{n+1}\right\|_{\infty}^{2} \leq\left[1+\sigma^{2} \frac{t}{t+1}\right]^{n+1} E\left\|U_{m}^{0}\right\|_{\infty}^{2}, \\
& E\left\|U_{m}^{n+1}\right\|_{\infty} \leq\left[1+\sigma^{2} \frac{t}{t+1}\right]^{\frac{n+1}{2}} E\left\|U_{m}^{0}\right\|_{\infty}, \\
& E\left\|U^{n+1}\right\|_{\infty} \leq e^{\theta t} E\left\|U^{0}\right\|_{\infty},
\end{aligned}
$$

where $\theta=\frac{\sigma^{2}}{2}$. Therefore, according to Definition 5 , the proposed scheme is conditionally stable.

Theorem 9 If $\left(1+\sum_{k} A_{m k}-\sum_{k} B_{m k}\right) \leq 1$, then the SCFD scheme Eq. (7) with $(n+$ 1) $\Delta t=t$ is conditionally stable in the mean square with respect to the norm $|\cdot|_{2, \Delta x}=$ $\sqrt{\sum_{i=-\infty}^{\infty}|\cdot|^{2} \Delta x}$ for the SFADE. 
Proof Applying $E|\cdot|^{2}$ to Eq. (8) and using the independence of the Wiener increment, with $E\left[V_{n}\right]=0$ and $E\left[V_{n}^{2}\right]=1$, we obtain

$$
\begin{aligned}
& E\left|U_{m}^{n+1}\right|^{2}=E\left[U_{m}^{n}+\sum_{k} A_{m k} U_{k}^{n}-\sum_{k} B_{m k} U_{k}^{n}+\sigma \sqrt{\Delta t} V_{n} U_{m}^{n}\right]^{2}, \\
& E\left|U_{m}^{n+1}\right|^{2}=E\left|U_{m}^{n}+\sum_{k} A_{m k} U_{k}^{n}-\sum_{k} B_{m k} U_{k}^{n}\right|^{2}+\sigma^{2} \Delta t E\left|U_{m}^{n}\right|^{2} .
\end{aligned}
$$

Adding up over $m$ yields

$$
\sum_{m=-\infty}^{\infty} E\left|U_{m}^{n+1}\right|^{2} \Delta x \leq\left[S C^{2}+\sigma^{2} \Delta t\right] \sum_{m=-\infty}^{\infty} E\left|U_{m}^{n}\right|^{2} \Delta x,
$$

where

$$
S C=1+\sum_{k} A_{m k}-\sum_{k} B_{m k} .
$$

In case, $1+\sum_{k} A_{m k}-\sum_{k} B_{m k} \leq 1$, with $(n+1) \Delta t=t$, we have

$$
\begin{aligned}
& \sum_{m=-\infty}^{\infty} E\left|U_{m}^{n+1}\right|^{2} \Delta x \leq\left[1+\sigma^{2} \frac{t}{t+1}\right]^{n+1} \sum_{m=-\infty}^{\infty} E\left|U_{m}^{n}\right|^{2} \Delta x, \\
& E\left\|U_{m}^{n+1}\right\|_{2, \Delta x}^{2} \leq\left[1+\sigma^{2} \frac{t}{t+1}\right]^{n+1} E\left\|U_{m}^{n}\right\|_{2, \Delta x}^{2} \\
& E\left\|U_{m}^{n+1}\right\|_{2, \Delta x}^{2} \leq\left[1+\sigma^{2} \frac{t}{t+1}\right]^{n+1} E\left\|U_{m}^{n}\right\|_{2, \Delta x}^{2} \leq\left[1+\sigma^{2} \frac{t}{t+1}\right]^{n+1} E\left|U_{m}^{0}\right|_{2, \Delta x}^{2} .
\end{aligned}
$$

Since $\Delta t=\frac{t}{(n+1)}$, we get

$$
\begin{aligned}
& E\left\|U_{m}^{n+1}\right\|_{2, \Delta x}^{2} \leq\left[1+\sigma^{2} \frac{t}{t+1}\right]^{(n+1)} E\left\|U_{m}^{0}\right\|_{2, \Delta x^{\prime}}^{2} \\
& E\left\|U^{n+1}\right\|_{2, \Delta x}^{2} \leq e^{\theta t} E\left\|U^{0}\right\|_{2, \Delta x^{\prime}}
\end{aligned}
$$

where $\theta=\sigma^{2}$.

Therefore, according to Definition 5 , the proposed scheme is conditionally stable.

Theorem 10 SCFD scheme (7) is consistent in the mean square in the sense of Definition 1.

Proof Let $\Phi(x, t)$ be a smooth function, then we have

$$
\begin{aligned}
\left.\mathcal{L}(\Phi)\right|_{m} ^{n}= & \Phi(m \Delta x,(n+1) \Delta t)-\Phi(m \Delta x, n \Delta t) \\
& +\gamma \int_{n \Delta t}^{(n+1) \Delta t}{ }_{x} D_{\beta} \Phi(m \Delta x, s) d s-\rho \int_{n \Delta t}^{(n+1) \Delta t}{ }_{x} D_{\alpha} \Phi(m \Delta x, s) d s \\
& -\sigma \int_{n \Delta t}^{(n+1) \Delta t} \Phi(m \Delta x, s) d W(s),
\end{aligned}
$$


and

$$
\begin{aligned}
\mathcal{L}_{m}^{n}(\Phi)= & \Phi(m \Delta x,(n+1) \Delta t)-\Phi(m \Delta x, n \Delta t) \\
& +\gamma \Delta t \sum_{k=1} B_{m k} \Phi(m \Delta x, n \Delta t)-\rho \Delta t \sum_{k=1} A_{m k} \Phi(m \Delta x, n \Delta t) \\
& -\sigma \Phi(m \Delta x, n \Delta t)(W((n+1) \Delta t-W(n \Delta t)) .
\end{aligned}
$$

Note that here we have

$$
b^{\alpha}=\frac{1}{h^{\alpha} \Gamma(3-\alpha)}, \quad b^{\beta}=\frac{1}{h^{\beta} \Gamma(2-\beta)} .
$$

By the square property of Itô integral, we have

$$
\begin{aligned}
\mathbb{E}|\mathcal{L}(\Phi)|_{m}^{n}-\left.\mathcal{L}_{m}^{n}(\Phi)\right|^{2}= & \mathbb{E} \mid \gamma \int_{n \Delta t}^{(n+1) \Delta t}\left({ }_{x} D_{\beta} \Phi(m \Delta x, s)-\sum_{k=1} B_{m k} \Phi(m \Delta x, n \Delta t)\right) d s \\
& -\rho \int_{n \Delta t}^{(n+1) \Delta t}\left({ }_{x} D_{\alpha} \Phi(m \Delta x, s)-\sum_{k=1} A_{m k} \Phi(m \Delta x, n \Delta t)\right) d s \\
& -\left.\sigma \int_{n \Delta t}^{(n+1) \Delta t}(\Phi(m \Delta x, s)-\Phi(m \Delta x, n \Delta t)) d W(s)\right|^{2}
\end{aligned}
$$

we can use the following inequality:

$$
\begin{aligned}
& \mathbb{E}|X+Y+Z|^{2} \leq 4 \mathbb{E}|X|^{2}+4 \mathbb{E}|Y|^{2}+4 \mathbb{E}|Z|^{2}, \\
& \mathbb{E}|\mathcal{L}(\Phi)|_{m}^{n}-\left.\mathcal{L}_{m}^{n}(\Phi)\right|^{2} \\
& \leq 4(\gamma)^{2} \mathbb{E}\left|\int_{n \Delta t}^{(n+1) \Delta t}\left({ }_{x} D_{\beta} \Phi(m \Delta x, s)-\sum_{k=1} B_{m k} \Phi(m \Delta x, n \Delta t)\right) d s\right|^{2} \\
& \quad+4(\rho)^{2} \mathbb{E}\left|\int_{n \Delta t}^{(n+1) \Delta t}\left({ }_{x} D_{\alpha} \Phi(m \Delta x, s)-\sum_{k=1} A_{m k} \Phi(m \Delta x, n \Delta t)\right) d s\right|^{2} \\
& \quad+4(\sigma)^{2} \mathbb{E}\left|\int_{n \Delta t}^{(n+1) \Delta t}(\Phi(m \Delta x, s)-\Phi(m \Delta x, n \Delta t)) d W(s)\right|^{2} .
\end{aligned}
$$

Since $\Phi(x, t)$ is a deterministic function, then $\mathbb{E}|\mathcal{L}(\Phi)|_{m}^{n}-\left.\mathcal{L}_{m}^{n}(\Phi)\right|^{2} \rightarrow 0$ as $n, m \rightarrow \infty$. So, the SCFD scheme is consistent in the mean square.

\section{Numerical results}

In this section, the performance of the stochastic compact finite difference method (SCFDM) for solving the proposed stochastic fractional advection diffusion equation (SFADE) Eq. (1) is considered and applied to some test examples [47]. For computational purposes, it is useful to consider discrete Brownian motion where $W(t)$ is specified at discrete $t$ values. It is set 1000 code runs for each $\{\Delta x, \Delta t\}$ with different sample of noise by Matlab. 


\subsection{Example 1}

Examine the performance of the proposed SCFD schemes for stochastic diffusion equation of the form

$$
u_{t}(x, t)=\frac{1}{20} D_{x}^{\alpha} u(x, t)+\frac{5}{2} u(x, t) \dot{W}(t), \quad t \in\left(0, \frac{3}{5}\right], x \in(0,1),
$$

subject to the following initial condition:

$$
u(x, 0)=\exp \left(-\frac{\left(x-\frac{1}{2}\right)^{2}}{\frac{1}{20}}\right)
$$

with the boundary conditions

$$
\begin{aligned}
& u(0, t)=\frac{1}{\sqrt{4 t+1}} \exp \left(-\frac{\left(-\frac{1}{2}\right)^{2}}{\frac{1}{20}(4 t+1)}\right) \\
& u(1, t)=\frac{1}{\sqrt{4 t+1}} \exp \left(-\frac{\left(\frac{1}{2}\right)^{2}}{\frac{1}{20}(4 t+1)}\right)
\end{aligned}
$$

In the integer case $\alpha=2$, the exact solution is

$$
u(x, t)=\frac{1}{\sqrt{4 t+1}} \exp \left(-\frac{\left(x-\frac{1}{2}\right)^{2}}{\frac{1}{20}(4 t+1)}\right) .
$$

Let $N$ and $M$ be the total number of grid points for the space and time discretizations, respectively. We plot in Fig. 1 a solution of Eq. (15) using both the SCFD scheme along with the exact solution with $\alpha=2, N=100$, and $M=800$ at $t=\frac{3}{5}$. It can be seen that the numerical method scheme performs well in comparison with reference research.

Table 1 presents the absolute error of the solution of (15) using SCFDM during the time interval $\left[0, \frac{3}{5}\right]$ with $\alpha=2$ and different values of $M$ and $N$. We see that the SCFD method is stable and more accurate as $h, \Delta t \rightarrow 0$.

For studying the performance of the proposed SCFDM, we use the exact solution to evaluate the error function

$$
e_{i}^{n}=\left|u_{i}^{n}-u_{\text {exact }}(x, t)\right|
$$

Figure 1 The exact solution and a solution of SFDE using SCFDM

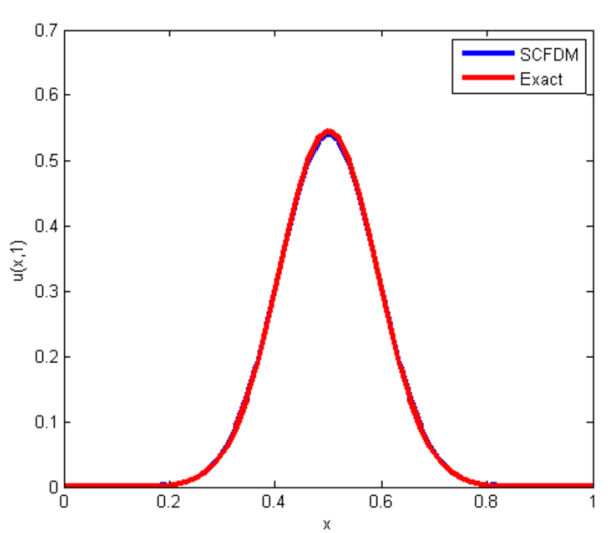


Table 1 The absolute error

\begin{tabular}{rrlll}
\hline$N$ & $M$ & $e(u(0.4,0.6))$ & $e(u(0.5,0.6))$ & $e(u(0.6,0.6))$ \\
\hline 25 & 50 & $3.9 \mathrm{E}-02$ & $1.5 \mathrm{E}-02$ & $2.5 \mathrm{E}-03$ \\
50 & 200 & $1.6 \mathrm{E}-02$ & $6.4 \mathrm{E}-03$ & $2.4 \mathrm{E}-03$ \\
100 & 800 & $3.9 \mathrm{E}-03$ & $2.2 \mathrm{E}-03$ & $3.2 \mathrm{E}-04$ \\
\hline
\end{tabular}

\begin{tabular}{rrll}
\hline$N$ & $M$ & $e(u(0.7,0.6))$ & $e(u(0.8,0.6))$ \\
\hline 25 & 50 & $2.7 \mathrm{E}-03$ & $6.2 \mathrm{E}-04$ \\
50 & 200 & $5.1 \mathrm{E}-04$ & $5.7 \mathrm{E}-04$ \\
100 & 800 & $1.4 \mathrm{E}-04$ & $1.2 \mathrm{E}-05$ \\
\hline
\end{tabular}

Table 2 Spatial and temporal error norms for SCFDM with $\alpha=2$ at $t=0.6$

\begin{tabular}{rrllll}
\hline$N$ & $M$ & $\|e\|_{L_{s}^{1}}$ & $\|e\|_{L_{s}^{2}}$ & $\|e\|_{L_{t}^{1}}$ & $\|e\|_{L_{t}^{2}}$ \\
\hline 25 & 50 & $3.8 \mathrm{E}-03$ & $8 \mathrm{E}-03$ & $3.8 \mathrm{E}-03$ & $5.1 \mathrm{E}-03$ \\
50 & 200 & $2.8 \mathrm{E}-03$ & $6.2 \mathrm{E}-03$ & $2.8 \mathrm{E}-03$ & $3.9 \mathrm{E}-03$ \\
100 & 800 & $1.3 \mathrm{E}-03$ & $2.4 \mathrm{E}-03$ & $1.4 \mathrm{E}-03$ & $1.2 \mathrm{E}-03$ \\
\hline
\end{tabular}

where $u_{\text {exact }}(x, t)$ and $u_{i}^{n}$ are respectively the exact and numerical solutions at the lattice points $\left(x_{i}, t_{n}\right)$.

The spatial discrete error norms are defined by

$$
\|e\|_{L_{S}^{1}}=h \sum_{i}\left|e_{i}\right|, \quad\|e\|_{L_{S}^{2}}=\left(h \sum_{i}\left|e_{i}\right|^{2}\right)^{1 / 2} .
$$

And to investigate the time accuracy of the method, we define the temporal discrete errornorms:

$$
\|e\|_{L_{t}^{1}}=\Delta t \sum_{n}\left\|e^{n}\right\|_{L_{s}^{2}}, \quad\|e\|_{L_{t}^{2}}=\left(\Delta t \sum_{n}\left\|e^{n}\right\|_{L_{s}^{2}}^{2}\right)^{1 / 2}
$$

where $\|\cdot\|_{L_{s}^{p}}=\|\cdot\|_{L^{p}([0, X])}$ and $\|\cdot\|_{L_{t}^{p}=\|\cdot\|_{L^{p}([0, T])}}$

denote the discrete $L^{p}$-norm in the space domain $(0, X)$ and the time interval $[0, T]$, respectively.

In Table 2, we summarize the spatial and temporal errors for the diffusion equation Eq. (15) with different values of $N$ and $M$ using the SCFD method. In comparison with the reference research, we can see the good performance of the SCFD scheme. A numerical solution of the stochastic diffusion equation Eq. (15) using the SCFD scheme is shown in Fig. 2 on time interval $\left[0, \frac{3}{5}\right]$ with $\alpha=2,1.6, N=100$, and $M=800$. We can see that the fractional diffusion $\alpha=1.6$ is slower than the standard diffusion $\alpha=2$.

Figure 3 shows the approximate solution of (15) obtained by SCFDM with different values of $\alpha, N=50$, and $M=200$ at time $t=0.6$. It can be observed that as $\alpha$ is decreased from 2 to 1 the amplitude of the solution behavior is increased. From Figs. 1-3 we conclude that the behavior of the solution continuously depends on the Caputo space fractional derivative. 

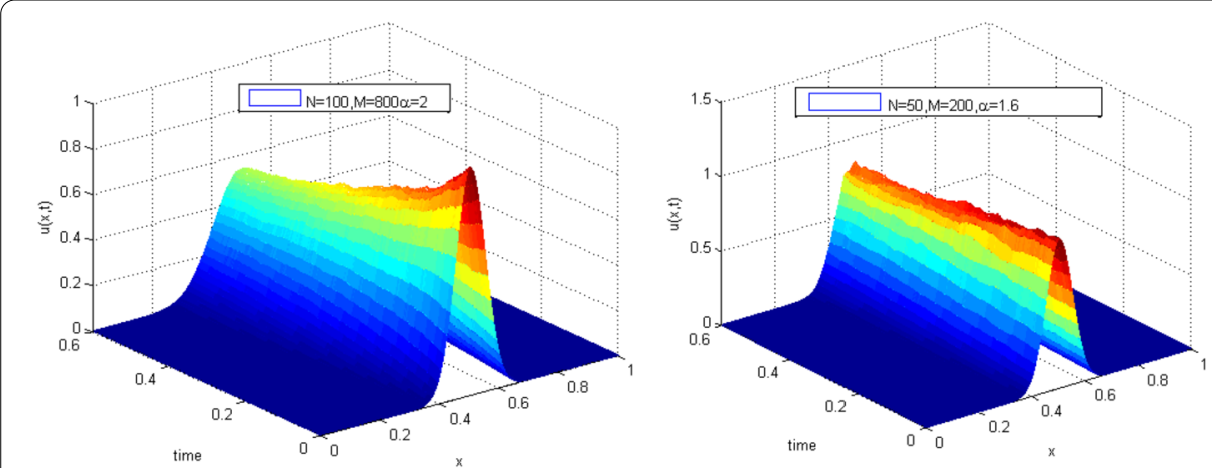

Figure 2 Mean solutions of stochastic diffusion equation using SCFDM

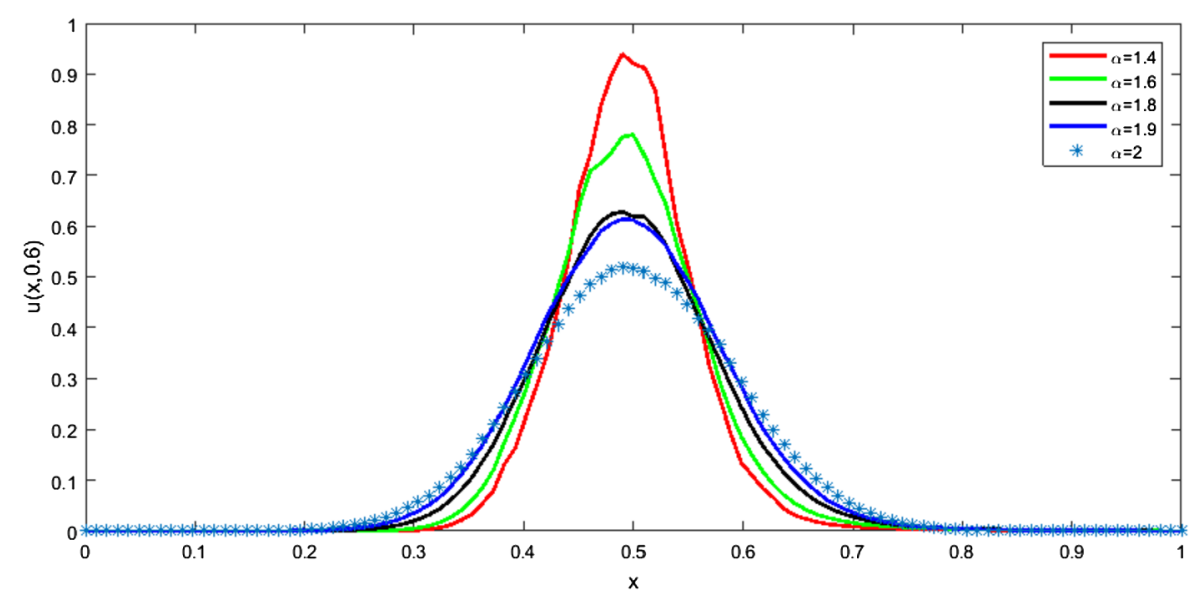

Figure 3 Mean solutions of SFDE using SCFDM

\subsection{Example 2}

In this example we investigate the efficiency of the SCFD scheme for approximating a solution of the SFADE of the form

$$
u_{t}(x, t)=\frac{1}{20} D_{x}^{\alpha} u(x, t)-\frac{1}{2} D_{x}^{\beta} u(x, t)+\frac{5}{2} u(x, t) \dot{W}(t), \quad t \in\left(0, \frac{3}{5}\right], x \in(0,1),
$$

subject to the following initial condition:

$$
u(x, 0)=\exp \left(-\frac{\left(x-\frac{1}{2}\right)^{2}}{\frac{1}{20}}\right)
$$

with the boundary conditions

$$
\begin{aligned}
& u(0, t)=\frac{1}{\sqrt{4 t+1}} \exp \left(-\frac{\left(-\frac{1}{2}-\frac{1}{2} t\right)^{2}}{\frac{1}{20}(4 t+1)}\right) \\
& u(1, t)=\frac{1}{\sqrt{4 t+1}} \exp \left(-\frac{\left(\frac{1}{2}-\frac{1}{2} t\right)^{2}}{\frac{1}{20}(4 t+1)}\right) .
\end{aligned}
$$


Figure 4 The exact solution and a solution of SFADE using SCFDM

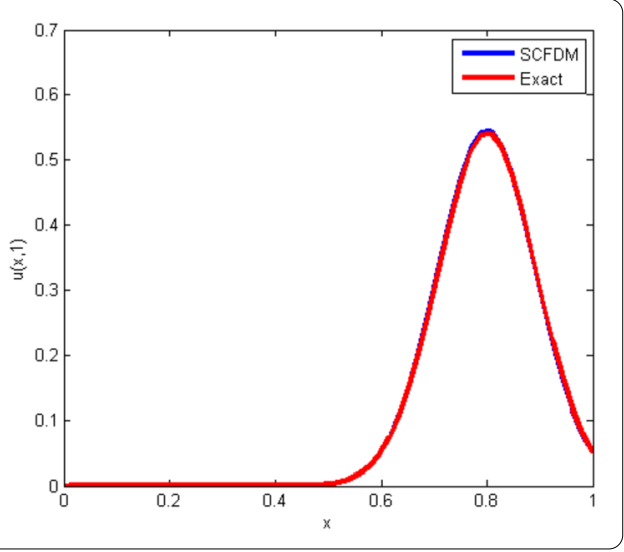

Table 3 The absolute error

\begin{tabular}{rllll}
\hline$N$ & $M$ & $e(u(0.4,0.6))$ & $e(u(0.5,0.6))$ & $e(u(0.6,0.6))$ \\
\hline 25 & 50 & $1.1 \mathrm{E}-04$ & $4.2 \mathrm{E}-04$ & $6 \mathrm{E}-03$ \\
50 & 200 & $1.4 \mathrm{E}-06$ & $1.2 \mathrm{E}-04$ & $5 \mathrm{E}-03$ \\
100 & 800 & $1.5 \mathrm{E}-07$ & $9.4 \mathrm{E}-05$ & $1.9 \mathrm{E}-04$ \\
\hline
\end{tabular}

\begin{tabular}{rrll}
\hline$N$ & $M$ & $e(u(0.7,0.6))$ & $e(u(0.8,0.6))$ \\
\hline 25 & 50 & $1.3 \mathrm{E}-02$ & $2.2 \mathrm{E}-03$ \\
50 & 200 & $4.4 \mathrm{E}-03$ & $1.9 \mathrm{E}-03$ \\
100 & 800 & $1.8 \mathrm{E}-03$ & $8.7 \mathrm{E}-04$ \\
\hline
\end{tabular}

Table 4 Spatial and temporal error norms for SCFDM with $\alpha=2$ and $\beta=1$ at $t=0.6$

\begin{tabular}{rrllll}
\hline$N$ & $M$ & $\|e\|_{L_{S}^{1}}$ & $\|e\|_{L_{S}^{2}}$ & $\|e\|_{L_{t}^{1}}$ & $\|e\|_{L_{t}^{2}}$ \\
\hline 25 & 50 & $9.3 \mathrm{E}-03$ & $1.7 \mathrm{E}-02$ & $7.5 \mathrm{E}-03$ & $1.02 \mathrm{E}-02$ \\
50 & 200 & $5.6 \mathrm{E}-03$ & $1.01 \mathrm{E}-02$ & $4.9 \mathrm{E}-03$ & $6.7 \mathrm{E}-03$ \\
100 & 800 & $1.2 \mathrm{E}-03$ & $2.6 \mathrm{E}-03$ & $1.3 \mathrm{E}-03$ & $1.7 \mathrm{E}-03$ \\
\hline
\end{tabular}

In the integer, the exact solution is

$$
u(x, t)=\frac{1}{\sqrt{4 t+1}} \exp \left(-\frac{\left(x-\frac{1}{2}-\frac{1}{2} t\right)^{2}}{\frac{1}{20}(4 t+1)}\right)
$$

Figure 4 shows a solution of (16) using both the SCFD scheme along with the exact solution with $\alpha=2, \beta=1, N=100$, and $M=800$ at time $\frac{3}{5}$. We can see the good performance of the SCFD method.

In Table 3, the absolute error of the solution of (16) using SCFDM during the time interval $\left[0, \frac{3}{5}\right]$ with $\alpha=2, \beta=1$ and different values of $M$ and $N$ is given.

Table 4 shows the spatial and temporal errors for SFADE Eq. (16) with several values of $N$ and $M$ using the SCFDM. The results in Tables 3 and 4 show that the SCFD scheme performs better than the reference research.

Figure 5 shows the approximations of SFADE Eq. (16) using the SCFD scheme on a 100 by 800 grid during the time interval $[0,0.6]$.

From the figures we see that as $\alpha$ and $\beta$ are decreased, the amplitude of the solution behavior is increased. 

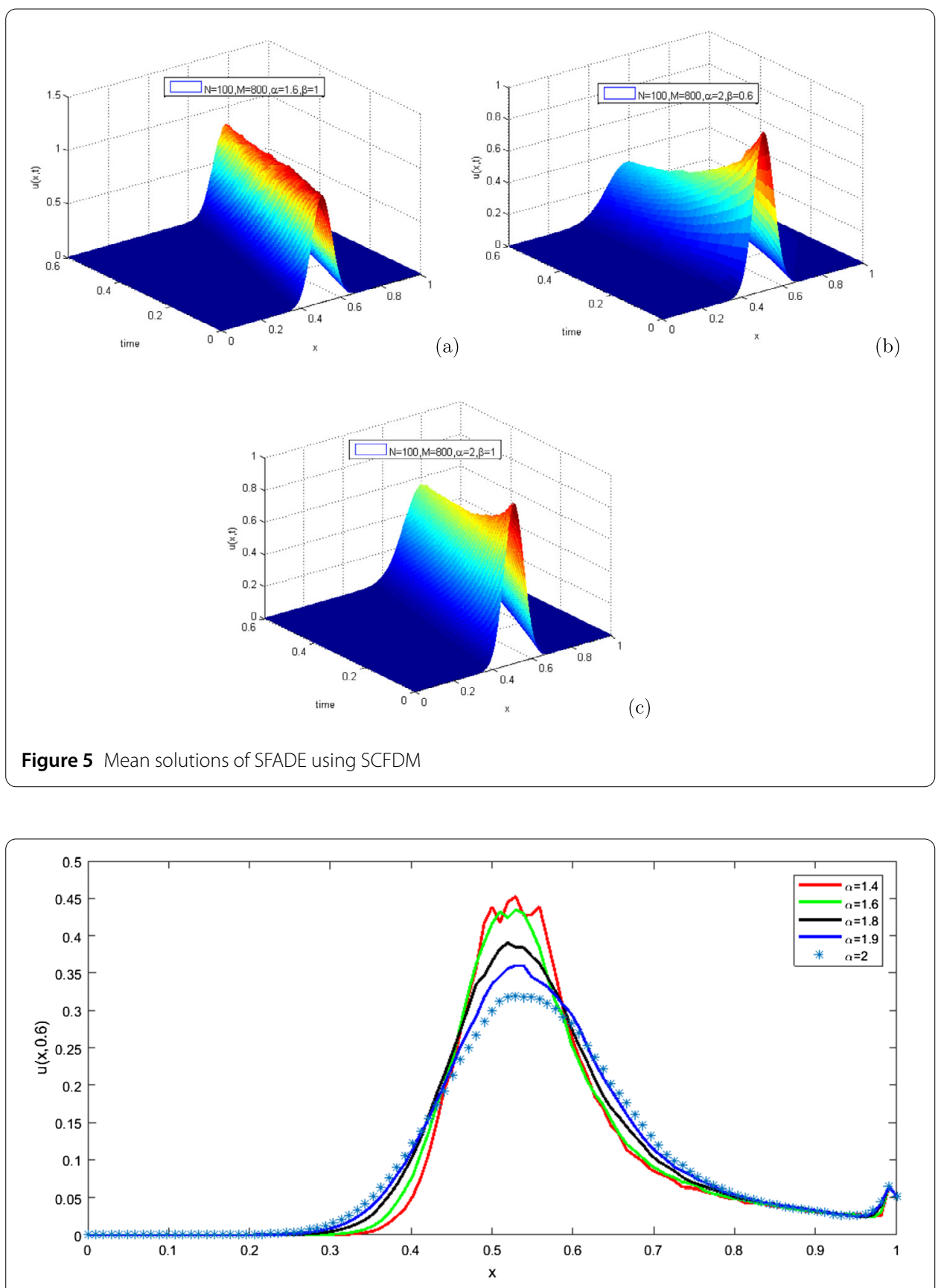

Figure 6 An approximate solution with $\beta=0.4$ and $\alpha=1.4,1.6,1.8,1.9,2$

Figure 6 shows an approximate solution obtained of SFADE Eq. (16) by SCFDM for different values of $\alpha$ and $\beta=0.4$ on a mesh of 100 and 800 grid points. It can be seen that the process described by fractional $\alpha$ is slightly more skewed to the left than that modeled by the standard $\alpha=2$.

Figure 7 shows an approximate solution of SFADE Eq. (16) obtained by SCFDM for different values of $\beta$ and $\alpha=1.8$ on a mesh of 100 and 800 grid points. It can be seen that the process described by fractional $\beta$ is also slightly more skewed to the left than that modeled by the standard $\beta=1$. 


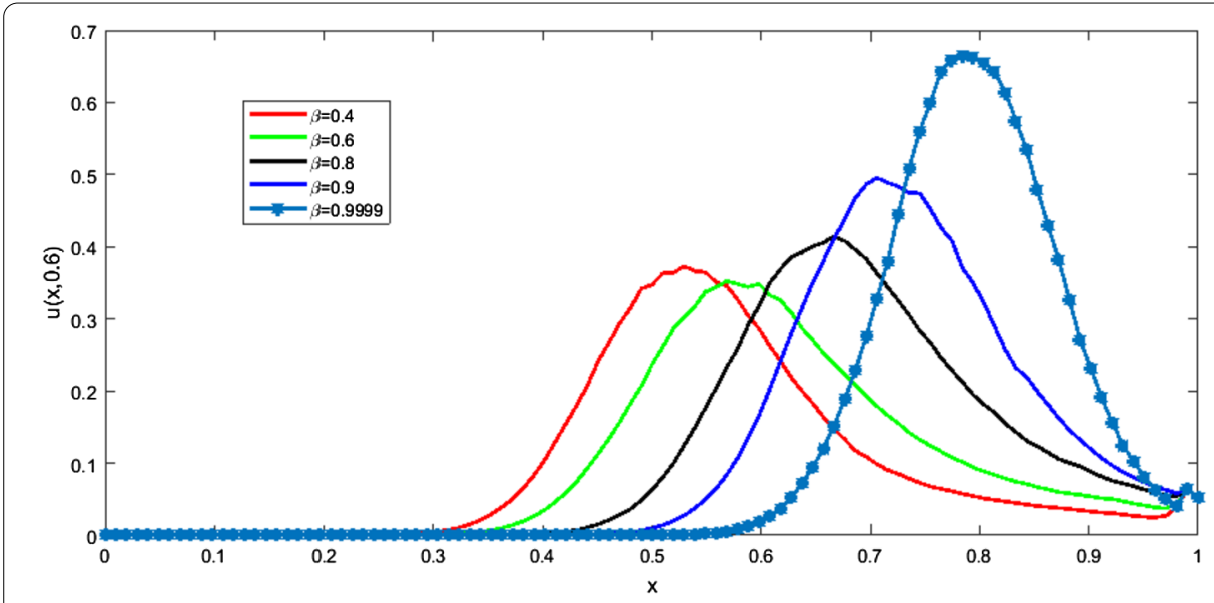

Figure 7 An approximate solution with $\alpha=1.8$ and $\beta=0.4,0.6,0.8,0.9,0.9999$

From Figs. 4-7, we conclude that the solution when $\alpha$ and $\beta$ are fractional orders is slower than the solution in the integer case when $\alpha=2, \beta=1$. Furthermore, it is seen that the solution continuously depends on the Caputo space fractional derivatives.

\section{Conclusions}

In this paper, a numerical solution for SFADE with real-valued Brownian motion is studied using effective SCFDM. The stability and consistency of the SCFD scheme are investigated for the SFADE with respect to a norm in the mean square, where the scheme is conditionally stable. Some numerical results and figures are given to demonstrate that the SCFDM performs well in approximating the exact solution of the SFADE and how it is computationally applicable.

Acknowledgements

The authors would like to thank the authors of the research in reference [9] and of the book in reference [44], they greatly helped in the mathematical derivation in the paper.

Funding

This research received no specific grant from any funding agency in the public, commercial, or not-for-profit sectors.

Availability of data and materials

Please contact the corresponding author for data requests.

Competing interests

The authors declare that they have no competing interest.

Authors' contributions

All authors contributed equally to this work. All authors read and approved the final manuscript.

\section{Publisher's Note}

Springer Nature remains neutral with regard to jurisdictional claims in published maps and institutional affiliations.

Received: 12 January 2020 Accepted: 16 April 2020 Published online: 29 April 2020

\section{References}

1. Prato, G.D., Tubaro, L.: Stochastic Partial Differential Equations and Application. Longman, Harlow (1992)

2. Gyongy, l.: Lattice approximations for stochastic quasi-linear parabolic partial differential equation driven by space-time white noise II. Potential Anal. 11, 1-37 (1999)

3. Gyongy, I., Rovira, C.: On $L^{P}$-solution of semilinear stochastic partial differential equation. Stoch. Process. Appl. 90, 83-108 (2000) 
4. Gyongy, l.: Existence and uniqueness results for semilinear stochastic partial differential. Stoch. Process. Appl. 73, 271-299 (1998)

5. Allen, E.J., Novosel, S.J., Zhang, Z.C.: Finite element and difference approximation of some linear stochastic partial differential equations. Stoch. Stoch. Rep. 64, 117-142 (1998)

6. Walsh, J.B.: Finite element methods for parabolic stochastic PDE's. Potential Anal. 23, 1-43 (2005)

7. Deb, M.K., Babuska, I.M., Oden, J.T.: Solution of stochastic partial differential equation using Galerkin finite element techniques. Comput. Methods Appl. Mech. Eng. 190, 6359-6372 (2001)

8. Sweilam, N.H., ElSakout, D.M., Muttardi, M.M.: High-resolution schemes for stochastic nonlinear conservation laws. Int. J. Appl. Comput. Math. 6, 22 (2020)

9. Roth, C.: Difference methods for stochastic partial differential equations. Z. Angew. Math. Mech. 82, 821-830 (2002)

10. Soheili, A.R., Niasar, M.B., Arezoomandan, M.: Approximation of stochastic parabolic differential equations with two different finite difference scheme. Bull. Iran. Math. Soc. 37, 61-83 (2011)

11. Kamrani, M., Hosseini, S.M.: The role of the coefficients of a general SPDE on the stability and convergence of the finite difference method. J. Comput. Appl. Math. 234, 1426-1434 (2010)

12. Kamrani, M., Hosseini, S.M.: Spectral collocation method for stochastic Burgers equation driven by additive noise. Math. Comput. Simul. 82, 1630-1644 (2012)

13. Ashyralyev, A.: On modified Crank-Nicolson differential scheme for stochastic parabolic equation. Numer. Funct. Anal. Optim. 29, 268-282 (2008)

14. Caputo, M.: Diffusion with space memory modelled with distributed order space fractional differential equations. Ann. Geophys. 46, 223-234 (2003)

15. Sokolov, I.M., Chechkin, A.V., Klafter, J.: Distributed-order fractional kinetics. Acta Phys. Pol. 35, 1323-1341 (2004)

16. Doha, E.H., Abd-Elhameed, W.M., Elkot, N.A., Youssri, Y.H.: Integral spectral tchebyshev approach for solving space Riemann-Liouville and Riesz fractional advection-dispersion problems. Adv. Differ. Equ. 2017, 284 (2017)

17. Hafez, R.M., Jacobi, Y.H.Y.: Collocation scheme for variable-order fractional reaction-subdiffusion equation. Comput. Appl. Math. 37, 5315-5333 (2018)

18. Doha, E.H., Youssri, Y.H.: On the connection coefficients and recurrence relations arising from expansions in series of modified generalized Laguerre polynomials: applications on a semi-infinite domain. Nonlinear Eng. 8, 318-327 (2019)

19. Raja, M.A.Z., Umar, M., Sabir, Z., Khan, J.A., Baleanu, D.: A new stochastic computing paradigm for the dynamics of nonlinear singular heat conduction model of the human head. Eur. Phys. J. Plus 2018, 133 (2018)

20. Amiri, S., Hajipour, M., Baleanu, D.: On accurate solution of the Fredholm integral equations of the second kind. Appl. Numer. Math. 150, 478-490 (2020)

21. Anguraj, A., Ravikumar, K., Baleanu, D.: Approximate controllability of a semilinear impulsive stochastic system with nonlocal conditions and Poisson jumps. Adv. Differ. Equ. 2020, 65 (2020)

22. Raza, A., Rafiq, M., Baleanu, D., Arif, M.S., Naveed, M., Ashraf, K.: Competitive numerical analysis for stochastic HIV/AIDS epidemic model in a two-sex population. IET Syst. Biol. 13, 305-315 (2019)

23. West, B.J., Grigolini, P., Metzler, R., Nonnenmacher, T.F.: Fractional diffusion and Lévy stable processes. Phys. Rev. E 55 99-106 (1997)

24. Metzler, R., Klafter, J.: The random walk's guide to anomalous diffusion: a fractional dynamics approach. Phys. Rep. 339, 1-77 (2000)

25. Chen, W.: A speculative study of 2/3-order fractional Laplacian modeling of turbulence: some thoughts and conjectures. AlP Chaos 16, 1-6 (2006)

26. Weron, K., Kotulski, M.: On the Cole-Cole relaxation function and related Mittag-Leffler distribution. Physica A 232 180-188 (1996)

27. Paradisi, P., Cesari, R., Mainardi, F., Tampieri, F.: The fractional Fick's law for non-local transport processes. Physica A 293, 130-142 (2001)

28. Baeumer, B., Kurita, S., Meerschaert, M.M.: Inhomogeneous fractional diffusion equations. Fract. Calc. Appl. Anal. 8 371-386 (2005)

29. Sun, H., Chen, Y., Chen, W.: Random-order fractional differential equation models. Signal Process. 91, 525-530 (2011)

30. Li, Q., Zhou, Y., Zhao, X., Ge, X.: Fractional order stochastic differential equation with application in European option pricing. Discrete Dyn. Nat. Soc. 12, 1-12 (2014)

31. Kamrani, M.: Numerical solution of stochastic fractional differential equations. Numer. Algorithms 68, 81-93 (2015)

32. Namjoo, M., Mohebbian, A.: Approximation of stochastic advection-diffusion equations with finite difference scheme. J. Math. Model. 4, 1-18 (2016)

33. Rubbab, Q., Mirza, I.A., Qureshi, M.Z.A.: Analytical solutions to the fractional advection-diffusion equation with time-dependent pulses on the boundary. AlP Adv. 6, 1-11 (2016)

34. Liu, F., Anh, V., Turner, I., Zhuang, P.: Time fractional advection dispersion equation. J. Appl. Math. Comput. 13, 233-245 (2003)

35. Huang, F., Liu, F.: The fundamental solution of the space-time fractional advection-dispersion equation. J. Appl. Math. Comput. 19, 233-245 (2005)

36. Meerschaert, M., Tadjeran, C.: Finite difference approximations for fractional advection-dispersion flow equations. J. Comput. Appl. Math. 172, 65-77 (2004)

37. Hou, T.Y., Luo, W., Rozonski, B., Zhou, H.M.: Wiener chaos expansion and numerical solution of randomly forced equations of fluid mechanics. J. Comput. Phys. 216, 687-706 (2006)

38. Ancey, C., Bohorquez, P., Heyman, J.: Stochastic interpretation of the advection-diffusion equation and its relevance to bed load transport. LGR 1002, 2529-2551 (2014)

39. Bishehniasar, M., Soheili, A.R.: Approximation of stochastic advection diffusion equation using compact finite difference technique. Iran. J. Sci. Technol. Trans. A, Sci. 37, 327-333 (2013)

40. Kloeden, P.E., Platen, E.: Numerical Solution of Stochastic Differential Equations. Springer, Berlin (1995)

41. Samko, S.G., Kilbas, A.A., Marichev, O.I.: Fractional Integrals and Derivatives: Theory and Applications. Gordon \& Breach, Philadelphia (1993)

42. Podlubny, l.: Fractional Differential Equations. Academic Press, New York (1999) 
43. Thomas, J.W.: Numerical Partial Differential Equations: Finite Difference Methods. Springer, Berlin (1998)

44. Lord, G.J., Powell, C.E., Sharlow, T.: An Introduction to Computational Stochastic PDES. Cambridge Texts in Applied Mathematics (2014)

45. Higham, D.J.: A-stability and stochastic mean-square stability. BIT Numer. Math. 40, 404-409 (2000)

46. Strikwerda, J.C.: Finite Difference Schemes and Partial Differential Equations, 2nd edn. SIAM, Philadelphia (2004)

47. Soheili, A.R., Mashad, M., Arezoomandan, Z.: Approximation of stochastic advection-diffusion equation with stochastic alternating direction explicit methods. Appl. Math. 58, 439-471 (2013)

48. Sweilam, N.H., Ahmed, S.M., Adel, M.: A simple numerical method for two-dimensional nonlinear fractional anomalous sub-diffusion equations. Math. Meth. Appl. Sci. 10 (2020)

49. Li, C., Zhao, Z., Chen, Y.: Numerical approximation of nonlinear fractional differential equations with subdiffusion and superdiffusion. Comput. Math. Appl. 62, 855-875 (2011)

50. Meerschaert, M.M., Tadjeran, C.: Finite difference approximations for fractional advection-dispersion flow equations. Comput. Math. Appl. 172, 65-77 (2004)

51. Shen, S., Liu, F.: Error analysis of an explicit finite difference approximation for the space fractional diffusion equation with insulated ends. ANZIAM J. 40, 871-887 (2000)

52. Yang, Q., Liu, F., Turner, I.: Numerical methods for fractional partial differential equations with Riesz space fractional derivatives. Appl. Math. Model. 34, 200-218 (2010)

\section{Submit your manuscript to a SpringerOpen ${ }^{\circ}$} journal and benefit from:

- Convenient online submission

Rigorous peer review

Open access: articles freely available online

High visibility within the field

- Retaining the copyright to your article

Submit your next manuscript at $\boldsymbol{~ s p r i n g e r o p e n . c o m ~}$ 